\title{
Reduced prefrontal activation during performance of the Iowa Gambling Task in
}

patients with bipolar disorder

Yasuki Ono $^{\mathrm{a}, \mathrm{b},{ }^{*}}$, Mitsuru Kikuchi ${ }^{\mathrm{a}, \mathrm{c}}$, Tetsu Hirosawa ${ }^{\mathrm{a}}$, Shoryoku Hino ${ }^{\mathrm{d}}$, Tatsuya

Nagasawa $^{\mathrm{a}}$, Takanori Hashimoto ${ }^{\mathrm{a}}$, Toshio Munesue ${ }^{\mathrm{a}, \mathrm{c}}$, Yoshio Minabe $\mathrm{a}^{\mathrm{a}, \mathrm{c}}$

${ }^{a}$ Department of Psychiatry and Neurobiology, Graduate School of Medical Science,

Kanazawa University, Kanazawa, Japan

${ }^{\mathrm{b}}$ Department of Psychiatry, Komatsu City Hospital, Komatsu, Japan

${ }^{c}$ Research Centre for Child Mental Development, Kanazawa University, Kanazawa,

Japan

${ }^{\mathrm{d}}$ Department of Neuropsychiatry, Ishikawa Prefectural Takamatsu Hospital, Ishikawa,

Japan

*Corresponding author: Department of Psychiatry, Komatsu City Hospital, ho 60,

Mukaimotoorimachi, Komatsu, Ishikawa 923-8560, Japan; Tel.: +81 761 22 7111; Fax:

+81761227199.

E-mail address: spfy7ff9@wish.ocn.ne.jp 


\section{Abstract}

The Iowa Gambling Task (IGT) is a complex decision-making task in which monetary wins and losses guide the development of strategies. The objective of this study was to evaluate hemodynamic responses of patients with bipolar disorder (BD) during performance of the IGT using near-infrared spectroscopy (NIRS). Participants comprised 13 patients and 15 healthy control subjects who were matched for age, sex, handedness, and intelligence quotient. Relative changes in oxygenated and deoxygenated hemoglobin (oxy-Hb and deoxy-Hb) levels in the frontal region were measured using a 46-channel NIRS system. All subjects were evaluated using NIRS during a verbal fluency task (VFT) and the IGT. During performance of the IGT, BD patients showed significantly decreased oxy-Hb levels in the bilateral orbitofrontal cortex (OFC) and left prefrontal cortex (PFC) compared with normal control subjects.

However, during the VFT, patients with BD showed no significant changes in oxy- $\mathrm{Hb}$ levels compared with control subjects. Changes in oxy-Hb levels in the bilateral OFC and the PFC during the IGT were negatively correlated with total scores on the Hamilton Rating Scale for Depression (HAM-D). Although the IGT was useful for differentiating patients with BP from control subjects, no significant differences in autonomic activity were observed. 
Keywords: Bipolar disorder, Near-infrared spectroscopy, Autonomic activity 


\section{1, Introduction}

Mood disorders, such as major depressive disorder (MDD) and bipolar disorder

(BD), have been evaluated using near-infrared spectroscopy (NIRS) during the

performance of various tasks, including a verbal fluency task (VFT; Kameyama et al.,

2006; Matsuo et al., 2007; Kubota et al., 2009; Noda et al., 2012) and a working

memory task (Schecklmann et al., 2011). Compared with functional magnetic

resonance imaging (fMRI), NIRS does not detect changes in deep brain structures and

has inferior spatial resolution, although it has fewer restrictions. In particular, NIRS is

less sensitive to motion artifacts, and due to the absence of magnetic effects of computer

and monitors, it can be applied even to newborn babies.

$\mathrm{BD}$ is a chronic and debilitating mood disorder that is associated with substantial

morbidity and mortality (Oquendo et al., 2000), with odds ratios for suicide attempts of

6.2 among patients with BD and 3.1 among patients with MDD (Chen et al., 1996).

Thus, clinical evaluations of impulsivity are critical in patients with BD.

The Iowa Gambling Task (IGT) is a prototypic tool for the investigation of processes that underpin incentive-related decision-making. It is a clinically sensitive tool that emulates real-world financial decision-making by requiring participants to select cards arranged in four decks. Each card has a monetary value that is revealed only after 
selection, and can either be a gain or a loss. The aim of the task is to optimize net gains across several trials. Two decks have higher rewards (gains) but also higher risks (losses) and can result in disadvantageous monetary losses over time. The other two decks have lower rewards and risks, making them advantageous in the long term. Differences in IGT performance have been observed in individuals with neuropsychiatric disorders that are characterized by problems in impulse control and emotion regulation.

Several fMRI studies have used the IGT as a measure of impulsivity (Lawrence et al., 2009; Hartstra et al., 2010; Li et al., 2010). Only one study, however, has reported fMRI and blood oxygen measurements during performance of the IGT in patients with BD (Frangou et al., 2008). In that study, patients with BD showed attenuated activation in both ventral and dorsal prefrontal cortices (PFCs) and increased activity in the lateral temporal and polar regions.

The somatic marker hypothesis (SMH; Damasio, 1996) provides a relevant theoretical framework for studying decision-making processes and the role of psychophysiological reactions in the anticipation of rewards or losses. It postulates that unconscious bodily states ("somatic markers") guide our behavior. In particular, pathological gamblers show decreases in heart rate (HR) after losses and wins. 
Although the absence of HR increases after wins indicates that reward sensitivity is decreased in pathological gamblers (Goudriaan 2006), no previous reports have evaluated the SMH in patients with BD.

The aim of the present study was to evaluate PFC activation during the IGT and the VFT, which require differing brain activations. Compared with the VFT, the IGT demonstrates impulsivity and presumably relies more on attentional and working memory processes. It may help to differentiate BD patients from healthy controls. A second aim was to determine whether sympathetic and parasympathetic activation showed changes in BD patients during performance of the IGT.

\section{2, Methods}

\subsection{Subjects}

Twenty-eight individuals were included in this study (see Table 1). These included 13 patients with BP-II disorders during euthymic or subdepressive episodes and 15 healthy controls. Premorbid intelligence quotient (IQ) was estimated using the Japanese version of the National Adult Reading Test (Matsuoka et al., 2006). Executive functions were evaluated according to the Behavioral Assessment of the Dysexecutive Syndrome (BADS; Wilson et al., 1996). Participants were assessed as being right-handed according to the Edinburgh Handedness Index if they used their right hand 
for $>85 \%$ of items (Oldfield, 1971). All subjects were right-handed according to the Edinburgh Handedness Index and were native speakers of Japanese.

Diagnoses were performed by experienced psychiatrists and were confirmed using the Structured Clinical Interview for DSM-IV (SCID-I). Patients ranged in age from 19 to 45 years. Subjects with a history of head injury or neurological disorders were excluded. None of the included patients received electroconvulsive therapy in the 3 months before the study. However, two patients suffered from co-morbid panic disorder, one suffered from social anxiety disorder, and one suffered from gender identity disorder.

At the time of testing, 11 patients were receiving neuroleptic medications; all were receiving lithium, valproate alone or a combination of these medications; one patient was receiving a selective serotonin reuptake inhibitor (SSRI); and eight patients were receiving second-generation antipsychotics.

Clinical ratings were assessed using the Hamilton Rating Scale for Depression (HAM-D; Hamilton 1960) and the Young Mania Rating Scale (YMRS; Young et al., 1978).

Fifteen healthy volunteers (23-43 years of age) were recruited as controls after they responded to an advertisement in Kanazawa. Control subjects had no history of psychiatric or neurological disorders and no first-degree relatives with bipolar diagnoses. 
They were not taking any drugs and were interviewed with the SCID-I. Table 1

summarizes the demographic characteristics of the patients and controls. The patients did not differ significantly in age, gender, handedness, estimated premorbid IQ, or

BADS.

The study was approved by the local research ethics committees, and after complete disclosure of the study details, all participants gave written informed consent. Upon completion of the experiment, all participants received 5000 yen as compensation for participation.

\subsection{Task procedure}

\subsubsection{VFT}

A letter version of the VFT was used as the target task, and the word repetition task (WRT) was used as the control task (CT). Subjects were asked to generate as many words as possible using the initial syllables /te/, /i/, or /shi/ in this order.

Three repetitions of VFT and WRT tasks were conducted in the following order: WRT, VFT, WRT, VFT, WRT, VFT, and WRT. The duration of the WRT was $80 \mathrm{~s}$ and that of the VFT was $60 \mathrm{~s}$. Subjects were instructed to repeat "'hai " during the WRT, which means "yes" in Japanese. 


\subsubsection{IGT}

A computerized version of the IGT (Version 2.0, 2002, Antoine Bechara) was used.

Subjects were instructed to select 100 cards arranged in four decks with the intention of gaining simulated monetary rewards. In each trial, subjects used a mouse to select cards from one of the four decks; the amounts won or lost in the trial, and total cumulative earnings were recorded. After NIRS measurements, subjects continued to play until the game had been completed.

Unknown to the subjects, deck A had frequent small punishments, deck B had infrequent but larger punishments, deck $\mathrm{C}$ had frequent small rewards, and deck D had infrequent large rewards. Subjects were asked to select a card from a deck of their choice taking their own time. The control task (CT) was designed to match the sensorimotor demands of the IGT, and subjects were instructed to select cards from the decks in the sequence A-, B-, C-, D-, A-, B-, C-, D. Both the IGT and the CT were performed for $60 \mathrm{~s}$. Five repetitions of the IGT and six repetitions of the CT were conducted in the following order: CT, IGT, CT, IGT, CT, IGT, CT, IGT, CT, IGT, and CT (Fig. 1).

Performance was measured by the net global outcome scores (net scores), which were calculated by subtracting the total number of cards selected from the disadvantage decks 
$(A+B)$ from the total number of cards selected from the advantage decks $(C+D)$.

\subsection{NIRS measurements}

A 46-channel NIRS imaging system (ETG-4000; Hitachi Medical Corporation, Tokyo, Japan) was used to measure relative changes in oxygenated and deoxygenated hemoglobin (oxy- $\mathrm{Hb}$ and deoxy-Hb) levels at infrared wavelengths of 695 and $830 \mathrm{~nm}$ according to the modified Beer-Lambert law (Cope et al., 1988). This method allowed calculation of signals that reflected changes in arbitrary units of $\mathrm{Hb}$ (mM-mm; Maki et al., 1995).

Intensities of diodes were modulated at frequencies in the range of $1-10 \mathrm{kHz}$ to prevent cross-talk between channels and wavelengths. The distance between the injectors and the detectors was $30 \mathrm{~mm}$. A pair of three $\times$ three arrays with five injectors and four detectors (probe 1), and three $\times$ five arrays with eight injectors and seven detectors (probe 2) were placed bilaterally on the prefrontal and temporal surface regions (Figs.

$2 \mathrm{~A}$ and $\mathrm{B})$. The lowest probes were positioned along the Fp1-Fp2 line according to the international 10-20 system used in electroencephalographic measurements.

Correspondence between these NIRS channels and measurement points on the cerebral cortex was confirmed using a multi-subject study of anatomical cranio-cerebral 
correlations (Okamoto et al., 2004) and is presented according to the virtual registration method (Tsuzuki et al., 2007).

The sampling rate was $0.1 \mathrm{~s}$ and data were analyzed using the integral mode.

Subsequently, the sum and average block design data were calculated by fitting the baseline. The mean pre-task baseline was estimated over a 10 -s period immediately before the task period, and the mean post-task baseline was determined over the last 10 $\mathrm{s}$ of the post-task period. The data between these baselines were then fitted using a linear regression model. The moving average method was applied using a window width of $5 \mathrm{~s}$ to correct for short-term motion artifacts. Accordingly, parameters for the integral mode and waveforms of changes in oxy- $\mathrm{Hb}$, deoxy- $\mathrm{Hb}$ and total-Hb levels were acquired from each subject in all 46 channels.

The virtual registration method was used to map channels on certain brain areas that corresponded to regions of interest (ROIs; Tsuzuki et al., 2007). These ROIs were identified by correspondence between the NIRS channels and measurement points on the cerebral cortex according to the virtual registration method in which structural information from an anatomical database was used to obtain estimates of channel positions in a standardized stereotaxic 3D brain atlas (Okamoto et al., 2004). The PFC [Brodmann Area (BA) BA10/46], the temporal gyrus (TG; BA21/22), and the 
orbitofrontal cortex (OFC; BA 11) were identified as relevant ROIs (left PFC: ROI1, right PFC: ROI2, left TG: ROI3, right TG: ROI4, left OFC: ROI5, right OFC: ROI6;

Figs. 2B, C)

\subsection{Electrocardiogram (ECG) measurements during the IGT}

ECG measurements were amplified, digitized at a sampling rate of $200 \mathrm{~Hz}$, and recorded for offline analysis. R-peaks in the ECG waveform were detected using an automated detection algorithm and subsequently verified by visual inspection using a Hyper Wave 2.1 (Kissei Comtec, Nagano, Japan). Power spectral analysis of the beat-to-beat time series of R-R intervals was performed using a Hyper Wave 2.1 (Kissei

Comtec). Both low-frequency power (LF; $0.05-0.15 \mathrm{~Hz})$ and high-frequency power $(\mathrm{HF} ; 0.15-0.40 \mathrm{~Hz})$ were measured. Data from each band power were transformed into natural logarithms, and LF/HF ratios were calculated.

\subsection{Statistical analyses}

Chi-square tests and Student's $t$-tests were used to compare proportions and means, respectively, between the $\mathrm{BD}$ and control groups. Performance and numbers of cards selected in each block of the IGT were examined using two-way repeated measures analysis of variance (ANOVA). 
Changes in oxy-Hb levels between pre- and post-task baseline periods correlate more strongly with fMRI signals of blood-oxygenation levels than changes in deoxy-Hb levels (Strangman et al., 2002). Thus, assuming a more direct proxy for cognitive activation, the present NIRS analyses were focused on changes in oxy-Hb levels. Mean changes in oxy-Hb levels in each ROI during the IGT and the VFT were compared between the two groups using two-way repeated-measures ANOVA, followed by post hoc $t$-tests. To examine the changes in cortical activation in the left OFC during the IGT, changes in oxy-Hb levels over time were compared using one-way repeated measures ANOVA.

Receiver operating characteristic (ROC) curve tests for the differentiation of the BD and control groups were performed.

Comparisons of each physiological variable (LF, HF, LF/HF ratio) were made using two-way repeated-measures ANOVA (subject group $\times$ time point).

Finally, mean changes in oxy-Hb levels during the IGT and the VFT were correlated with HAM-D scores using Pearson correlation coefficients.

\section{Results}

\subsection{Behavioral data}

The number of words generated during the VFT differed significantly $(t=2.883$, 
$p=0.008)$ between the $\mathrm{BD}($ mean $\pm \mathrm{SD}: 8.2 \pm 2.6)$ and control groups (mean $\pm \mathrm{SD}$ :

$11.6 \pm 3.5)$. However, the total net scores of IGT did not differ significantly between

the groups $(t=0.902, p=0.378 ; \mathrm{BD}$, mean $\pm \mathrm{SD}:-4.3 \pm 13.0$; control, mean $\pm \mathrm{SD}: 3.1 \pm$ 27.8). To determine whether the net scores of each block differed between the groups, a subject group $(\mathrm{BD}$ vs. control $) \times$ block $(1-5)$ repeated measures ANOVA was

performed. The results revealed no significant group $\times$ block interactions $(F=1.969$,

$d f=4, p=0.1053)$, nor main effects for group $(F=0.785, d f=1, p=0.3844)$ but

showed a significant main effect for block $(F=2.760, d f=4, p=0.032$; Fig. 3$)$. The

total number of selected cards did not differ significantly $(t=1.149, p=0.26)$ between

the $\mathrm{BD}($ mean $\pm \mathrm{SD}: 84.5 \pm 8.5)$ and control (mean $\pm \mathrm{SD}: 80.9 \pm 7.1)$ groups. To

examine whether the number of cards taken from each block during the IGT differed

between the $\mathrm{BD}$ and control groups, subject group $(\mathrm{BD}$ vs. control $) \times$ block $(1-5)$

repeated measures ANOVAs were performed. The results revealed neither significant

group $\times$ block interactions $(F=1.306, d f=4, p=0.2735)$ nor a main effect for group $(F$

$=1.320, d f=1, p=0.2619)$ but showed a significant main effect for block $(F=12.504$,

$d f=4, p<0.0001)$

\subsection{Neural activity during IGT and VFT performance}

Grand-averaged waveforms across two or four channels in six regions are shown in Fig. 
4. Average changes in oxy-Hb levels during the last $30 \mathrm{~s}$ of each IGT and VFT task segment are shown in Fig. 5. Two-way repeated measures ANOVA of the IGT showed that the effect of group $(F=7.201, d f=1, p=0.013)$ was significant, but there were no significant effects of region $(F=0.744, d f=5, p=0.592)$ or region $\times$ group interactions $(F=1.408, d f=5, p=0.2266) . \quad$ The control group had significantly larger increases in oxy-Hb levels than the BD group in all ROIs except for the left $\mathrm{TG}(F=1.414, d f=$ $26, p=0.198)$, right TG $(F=1.165, d f=26, p=0.045)$, and right $\mathrm{PFC}(F=2.509, d f=$ $26, p=0.016$ ). Findings for the for IGT after Bonferroni correction for multiple comparisons were as follows: left PFC, $F=4.906, d f=19.705, p=0.003$; left OFC, $F=$ 2.043, $d f=24, p=0.003$; right $\mathrm{OFC}, F=1.040, d f=23, p=0.003$. Differences were considered significant when $p<0.008$.

Two-way repeated measures ANOVA of the VFT showed a significant main effect of region $(F=6.630, d f=5, p<0.001)$ and a significant interaction effect of region $\times$ group $(F=3.671, d f=5, p=0.0040)$, whereas the main effect of group $(F=2.902, d f=$ $1, p=0.1014$ ) was not significant. Post hoc $t$-tests were used to further analyze this interaction. The control group did not have significantly larger increases in oxy-Hb levels than those in the BD group in all ROIs. Findings for the VFT after Bonferroni correction for multiple comparison were as follows: left TG, $F=3.932, d f=26, p=$ 
0.026 ; right TG, $F=3.433, d f=26, p=0.053$; left $\mathrm{OFC}, F=0.001, d f=25, p=0.715$

right $\mathrm{OFC}, F=0.267, d f=24, p=0.163$; left PFC, $F=1.460, d f=26, p=0.754$; right

PFC, $F=1.440, d f=26, p=0.442 . \quad$ Differences were considered significant when $p$ $<0.008$.

Finally, we examined the changes in activation between the first and last blocks of the

IGT in the left OFC (Fig. 6). Comparison of changes in oxy-Hb levels in the left OFC

by one-way ANOVA revealed no significant changes over time in the control $(F=$

$0.989, d f=4, p=0.42)$ or the BD group $(F=0.725, d f=0.4, p=0.5784)$.

\subsection{ROC curve analysis}

The ROC curves of the IGT and the VFT in the left OFC are shown in Fig. 7.

The area under the curve (AUC) values for bilateral OFC and PFC were relatively

higher in the IGT than in the VFT (Table 2). The sensitivity and specificity of the IGT at the cut-off points for the left $\mathrm{OFC}$, right $\mathrm{OFC}$, left PFC, and right PFC were 80.0 and $72.7 \%, 85.7$ and $72.7 \%, 80.0$ and $76.9 \%$, and 86.7 and $84.6 \%$, respectively.

\subsection{HR variability}

No significant main effects of time course were observed in $\operatorname{LF}(F=2.335, d f=4, p=$

$0.610), \operatorname{HF}(F=0.403, d f=1, p=0.5315)$ or $\operatorname{LF} / \mathrm{HF}(F=0.303, d f=1, p=0.5871)$, and no significant differences between groups were observed in $\operatorname{LF}(F=0.078, d f=1, p=$ 
$0.7820), \mathrm{HF}(F=0.523, d f=4, p=0.7194)$ or $\mathrm{LF} / \mathrm{HF}$ ratios $(F=2.064, d f=4, p=$

0.0912) during each IGT block (Table 3). No interaction effects were observed between

group and time course $(\mathrm{LF}: F=1.633, d f=4, p=0.1723$; HF: $F=0.621, d f=4, p=$

0.6485; $\mathrm{LF} / \mathrm{HF}$ ratio: $F=0.893, d f=4, p=0.4710)$.

\subsection{Relationships between brain activation and HAM-D scores}

Correlations between HAM-D scores and changes in oxy-Hb levels in the six ROIs are shown in Table 4. HAM-D scores were correlated with changes in oxy-Hb levels in bilateral OFC and PFC during the IGT ( $r=-0.465$ to $-0.384, p=0.017-0.044)$.

However, during the VFT, HAM-D scores were correlated with changes in oxy-Hb levels only in right TG $(r=-0.439, p=0.02)$.

\section{Discussion}

In this study, we examined brain activation during performance of the IGT by measuring changes in regional cerebral blood volume using NIRS. The resulting data indicated prefrontal hypoactivation in patients with BD during the IGT in comparison with healthy control subjects. However, no significant differences in oxy-Hb levels between BD and control groups were observed during the VFT. Regional hemodynamic changes during the IGT in bilateral PFC and OFC were negatively 
correlated with HAM-D scores, and during the VFT, were negatively correlated only in right TG.

The present data do not indicate decreased sympathetic or parasympathetic activation in patients with $\mathrm{BD}$.

Several previous studies have evaluated hemodyamic changes during the IGT using positron emission tomography (PET) (Ernst et al., 2002; Bolla et al., 2004).

In the present study, control subjects had significantly larger increases in oxy- $\mathrm{Hb}$ levels in bilateral OFC and left PFC, but not in the right PFC and bilateral TG compared with patients with BD. We used the original version of the IGT without the recent modifications devised by Hartstra (2010) in which 16 trials are performed in a two-deck card game. Nonetheless, the analytical method remained very simple, and simple comparisons were made between active and control phases. These analyses resembled a previous PET study of sex-related differences in task performance and brain activity in the OFC and the dorsolateral PFC, which showed activation in extensive regions of right lateral $\mathrm{OFC}$, right dorsolateral $\mathrm{PFC}$, and left lateral $\mathrm{OFC}$ in men, and activation of left medial OFC in women (Bolla et al., 2004). Although the tasks were similar to those in the present study, PET sessions comprised six 1-min scans at 12-min intervals, whereas the present NIRS examinations were performed within 10 min. Hence, given 
similar male to female ratios, these differences in activation may reflect differences between PET and NIRS sessions. Moreover, NIRS is inferior in terms of spatial resolution, and activated regions tend to be diffuse and vague, potentially contributing to differing observations.

Hartstra et al. (2010) developed a simplified version of IGT in which reward rules had to be learned during acquisition of event-related fMRI data. In this study, lateral PFC activation steadily decreased over the course of the trials, and the lateral PFC was assumed to be responsible for general working memory. In the present study, hyperactivation did not change significantly during the task, suggesting a relationship with general working memory.

In the only previously published study of the IGT in patients with BD, Frangou et al. (2008) showed attenuated activation in both the ventral and the dorsal PFC. In contrast, our data show deactivation in vast regions of the frontal cortex. Whereas the present version of the IGT was self-paced and the CT entailed routine selection of cards in the same order, the CT in the study of Frangou et al. involved cognitive selection of cards as in the test, although subjects were informed that no money would be won or lost during this epoch. Factors that may result in the deactivation phenomena include the intention of the IGT to require a high working memory and consequent 
hypofrontality. Although cognitive tasks require various types of cognition, some demand higher working memory and others require attentiveness (Kubota et al., 2009). Callicott et al. (2003) demonstrated dorsolateral PFC hyperfrontality at lower working memory loads in patients with schizophrenia. However, hypofrontality was also observed upon breach of working memory capacity during the tasks. Hence, similar hypofrontality may have influenced the present observations. Task-induced deactivation (TID) may also cause decreases in oxy-Hb levels. Accordingly, in healthy subjects, deactivation of the hippocampus, amygdala, and medial OFC has been demonstrated during the IGT (Harstra et al., 2010; Li et al., 2010). These observations may reflect the default-mode network (DMN), which is believed to be associated with suppression of spontaneous brain activities and reallocation of resources to ongoing attention-demanding tasks (McKiernan et al., 2003). Moreover, patients with schizophrenia showed greater TID of the medial frontal cortex (Harrison et al., 2007). Because TID reflects elements of the DMN of brain functions that are associated with self-directed mental processes during rest (Raichle et al., 2001), TID may have led to hypoactivation to some extent in the present study. In remitted bipolar patients, relative deactivation within orbital and medial frontal cortices compared with controls was demonstrated during performance of the Stroop task (Kronhaus et al., 
2006). Resting state fMRI data showed decoupling of the dorsolateral PFC with the medial PFC (MPFC) in bipolar disorder and schizophrenia. However, positive correlations between the MPFC and the insula, and between the MPFC and the ventrolateral PFC, as found in the bipolar disorder group were not exhibited in the schizophrenia group (Chai et al., 2011). These differences may relate to the different levels of deactivation of two disorders.

Unlike the IGT, the VFT was not associated with differences between the control and BD groups. Moreover, only changes in oxy-Hb levels in the right TG were correlated with HAM-D scores. Noda et al. (2012) suggested that reduced right frontal temporal activation on NIRS during the VFT is related to individual HAM-D items in patients with MDD. Thus, the right temporal cortex may be marker of mood disorders. A previous NIRS study (Kameyama et al., 2006) reported that increases in oxy-Hb levels in patients with BD were smaller than those in healthy control subjects during the early period of the VFT and were greater during the late period of the task. However, other studies using VFT showed significantly diminished increases in oxy-Hb levels in patients with BD compared with control subjects (Matsuo et al., 2007). These inconsistencies may reflect the heterogeneity of patients with bipolar-I and -II disorders, varying mood states, and varying medications. Moreover, task procedures differed 
between these studies. Specifically, in the present study, the task was performed with three repetitions of the 60-s VFT, whereas in the study by Kameyama et al. (2006) assigned syllables were changed every $20 \mathrm{~s}$ during the 60 -s task period.

Although no previous studies report monitoring of HF and LF in BD subjects during the IGT, developmental analogues of the IGT have been used to show that impaired decision-making and attenuated sympathetic activation is associated with attention-deficit/hyperactivity disorder (ADHD) among boys (Bubier et al., 2008). It is generally accepted that the HF $(0.15-0.40 \mathrm{~Hz})$ component is mediated by cardiac parasympathetic tones. However, Moak et al. (2007) demonstrated that LF reflects baroreflex function rather than cardiac sympathetic innervations. In the present study, no differences in LF, HF or LF/HF ratios were observed during IGT. We only evaluated epochs of the IGT separately and did not compare risky and non-risky choices in detail. Thus, SMH cannot be ruled out on the basis of the present data.

The current study was limited by a small sample size, and all patients were actively taking psychiatric medications that may have affected the results. In particular, the antidepressant mirtazapine was previously shown to increase changes in oxy-Hb levels in healthy subjects (Kohmura et al., 2012). In a recent review article (Hafeman et al., 2012), secondary analyses of medication effects in fMRI and diffusion tensor imaging 
studies showed few significant effects of medications. However, longitudinal studies show normalizing effects of such medications. In addition, there were several comorbid disorders in the BD group, a factor that may have affected the results.

In this study, patient mood states ranged from euthymic to subclinically depressed.

Although BD was associated with state- and trait-related abnormalities of VPFC activation during an event-related color-naming Stroop interference task (Blumberg et al., 2003), further studies are required to confirm these differences.

In summary, IGT performance during NIRS differentiated between BD patients and healthy controls, and changes in oxy-Hb levels were correlated with HAM-D scores in the patients. Experiments with multiple tasks of differing requirements will facilitate future NIRS studies of mood disorders.

\section{Acknowledgements}

The authors thank Hitachi Medical Corporation for the use of the ETG-4000 instrument and for skilled technical support.

This research is partially supported by the Center of Innovation Program from the Japan Science and Technology Agency, JST. 


\section{References}

Blumberg, H.P., Leung, H.C., Skudlarski, P., Lacadie, C.M., Fredericks, C.A., Harris, B.C., Charney, D.S., Gore, J.C., Krystal, J.H., Peterson, B.S., 2003. A functional magnetic resonance imaging study of bipolar disorder: state- and trait-related dysfunction in ventral prefrontal cortices. Archives of General Psychiatry 60, 601-609.

Bolla, K., Eldreth, D., Matochik, J.A., Cadet, J.L., 2004. Sex-related differences in a gambling task and its neurological correlates. Cerebral Cortex 14, 1226-1232.

Bubier, J.L., Drabick, D.A.G., 2008. Affective decision making and externalizing behaviors: the role of autonomic activity. Journal of Abnormal Child Psychology 36, 941-953.

Callicott, J.H., Mattay, V.S., Verchinski, B.A., Marenco, S., Egan, M.F., Weinberger, D.R., 2003. Complexity of prefrontal dysfunction in schizophrenia: more than up or down. American Journal of Psychiatry 160, 2209-2215. 
Chai, X.J., Whitfield-Gabrieli, S., Shinn, A.K., Gabrieli, J.D.E, Castanon, A.N.,

McCarthy, J.M., Cohen, B.M., Ongur, D., 2011. Abnormal medial prefrontal cortex

resting-state connectivity in bipolar disorder and schizophrenia.

Neuropsychopharmacology 36, 2009-2017.

Chen, Y.W., Dilsaver, S.C., 1996. Life time rates of suicide attempts among subjects

with bipolar and unipolar disorders relative to subjects with other Axis 1 disorders.

Biological Psychiatry 39, 896-899.

Cope, M., Delpy, D.T., Reynolds, E.O., Wray, S., Wyatt, J., van der Zee, P., 1988.

Methods of quantitating cerebral near infrared spectroscopy data. Advances in

Experimental Medicine and Biology 222, 183-189.

Damasio, A.R., 1996. The somatic marker hypothesis and the possible functions of the prefrontal cortex. Philosophical Transactions of Royal Society London B:

Biological Sciences 351, 1413-1420.

Ernst, M., Bolla, K., Mouratidis, M., Contoreggi, C., Matochik, J.A., Kurian, V., Cadet, J.L., Kimes, A.S., London, E.D., 2002. Decision-making in a risk-taking task: a PET study. Neuropsychopharmacology 26, 682-691. 
Frangou, S., Kington, J., Raymont, V., Shergill, S., 2008. Examining ventral and dorsal prefrontal function in bipolar disorder: a functional magnetic resonance imaging study. European Psychiatry 23, 300-308.

Goudriaan, A.E., Oosterlaan, J., de Beurs, E., van den Brink, W., 2006.

Psychophysiological determinants and concomitants of deficient decision making in pathological gamblers. Drug and Alcohol Dependence 84, 231-239.

Hafeman, D.M., Chang, K.D., Garrett, A.S., Sanders, E.M., Phillips, M.L., 2012.

Effect of medication on neuroimaging findings in bipolar disorder: updated review. Bipolar Disorders 14, 375-410.

Hamilton, M., 1960. A rating scale for depression. Journal of Neurology, Neurosurgery, and Psychiatry 23, 56-62.

Harrison, B.J., Yucel, M., Pujol, J., Pantelis, C., 2007. Task-induced deactivation of midline cortical regions in schizophrenia assessed with fMRI. Schizophrenia Research $91,82-86$.

Hartstra, E., Oldenburg, J., Leijenhorst, L., Rombouts, S., Crone, E., 2010. Brain regions involved in the learning and application of reward rules in a two-deck gambling task. Neuropsychologia 48, 1438-1446. 
Kameyama, M., Fukuda, M., Yamagishi, Y., Sato, T., Uehara, T., Ito, M., Suto, T., Mikuni, M., 2006. Frontal lobe function in bipolar disorder: a multichannel near-infrared spectroscopy study. Neuroimage 29, 172-184.

Kohmura, K., Iwamoto, K., Aleksic, B., Sasada, K., Kawano, N., Katayama, H., Noda, A., Iidaka, T., Ozaki, N., 2012. Effect of sedative antidepressants on prefrontal cortex activity during verbal fluency task in healthy subjects: a near-infrared spectroscopy study. Psychopharmacology 226, 75-81.

Kronhaus, D.M., Lawrence, N.S., Williams, A.M., Frangou, S., Brammer, M.J., Williams, S.C.R., Andrew, C.M., Phillips, M.L., 2006. Stroop performance in bipolar disorder: further evidence for abnormalities in the ventral prefrontal cortex. Bipolar Disorders 8, 28-39.

Kubota, Y., Toichi, M., Shimizu, M., Mason, R., Findling, R., Yamamoto, K., Hayashi, T., Calabrese, J., 2009. Altered prefrontal lobe oxygenation in bipolar disorder: a study by near-infrared spectroscopy. Psychological Medicine 39, 1265-1275.

Lawrence, N., Jollant, F., O’Daly, O., Zelaya, F., Phillips, M., 2009. Distinct roles of prefrontal cortical subregions in the Iowa gambling task. Cerebral Cortex 19, 1134-1143. 
Li, X., Lu, Z.L., D’Argembeau, A., Ng, M., Bechara, A., 2010. The Iowa Gambling Task in fMRI images. Human Brain Mapping 31, 410-423.

Maki, A., Yamashita, Y., Ito, Y., Watanabe, E., Mayanagi, Y., Koizumi, H., 1995.

Spatial and temporal analysis of human motor activity using noninvasive NIR topography. Medical Physics 22, 1997-2005.

Matsuo, K., Kouno, T., Hatch, J.P., Seino, K., Ohtani, T., Kato, N., Kato, T., 2007. A near-infrared spectroscopy study of prefrontal cortex activation during a verbal fluency task and carbon dioxide inhalation in individuals with bipolar disorder. Bipolar Disorders 9, 876-883.

Matsuoka, K., Uno, M., Kasai, K., Koyama, K., Kim, Y., 2006. Estimation of premorbid IQ in individuals with Alzheimer's disease using Japanese ideographic script (Kanji) compound words: Japanese version of National Adult Reading Test. Psychiatry and Clinical Neuroscience 60 (3), 332-339.

McKiernan, K.A., Kaufman, J.N., Kucera-Thompson, J., Binder, J.R., 2003. A parametric manipulation of factors affecting task-induced deactivation in functional neuroimaging. Journal of Cognitive Neuroscience 15, 394-408. 
Moak, J.P., Goldstein, D.S., Eldadah, B.A., Saleem, A., Holmes, C., Pechnik, S., Sharabi, Y., 2007. Supine low-frequency power of heart rate variability reflects baroreflex, not cardiac sympathetic innervation. Heart Rhythm 4, 1523-1529.

Noda, T., Yoshida, S., Matsuda, T., Okamoto, N., Sakamoto, K., Koseki, S., Numachi, Y., Matsushima, E., Kunugi, H., Higuchi, T., 2012. Frontal and right temporal activations correlate negatively with depression severity during verbal fluency task: a multi-channel near-infrared spectroscopy study. Journal of Psychiatric Research 46, 905-912.

Okamoto, M., Dan, H., Sakamoto, K., Takeo, K., Shimizu, K., Kohno, S., Oda, I., Isobe, S., Suzuki, T., Kohyama, K., Dan, I., 2004. Three-dimensional probabilistic anatomical cranio-cerebral correlation via the international 10-20 system oriented for transcranial functional brain mapping. Neuroimage 21, 99-111.

Oldfield, R.C., 1971. The assessment and analysis of handedness: the Edinburgh Inventory. Neuropsychologia 9, 97-113.

Oquendo, M.A., Waternaux, C., Brodsky, B., Parsons, B., Haas, G.L., Malone, K.M., Mann, J.J., 2000. Suicidal behavior in bipolar mood disorder: clinical characteristics of attempters and nonattempters. Journal of Affective Disorders 59, 107-117. 
Raichle, M.E., MacLeod, A.M., Snyder, A.Z., Powers, W.J., Gusnard, D.A., Shulman, G.L., 2001. A default mode of brain function. Proceedings of the National Academy of Sciences of the United States of America 98, 676-682.

Schecklmann, M., Dresler, T., Beck, S., Jay, J., Febres, R., Haeusler, J., Jarczok, T., Reif, A., Plichta, M., Ehlis, A.C., Fallgatter, A., 2011. Reduced prefrontal oxygenation during object and spatial visual working memory in unipolar and bipolar depression. Psychiatry Research: Neuroimaging 194, 378-384.

Strangman, G., Boas, D.A., Sutton, J.P., 2002. Non-invasive neuroimaging using near-infrared light. Biological Psychiatry 52, 679-693.

Tsuzuki, D., Jurcak, V., Singh, A.K., Okamoto, M., Watanabe, E., Dan, I., 2007. Virtual spatial registration of stand-alone fNIRS data to MINI space. Neuroimage 34, $1506-1518$.

Young, R.C., Biggs, J.T., Ziegler, V.E., Meyer, D.A., 1978. A rating scale for mania: reliability, validity and sensitivity. British Journal of Psychiatry 133, 429-435.

Wilson, B.A., Alderman, N., Burgess, P.W., Emslie, H.E., Evans, J.J., 1996.

Behavioural Assessment of the Dysexecutive Syndrome. Thames Valley Test Company, Bury St Edmunds, UK. 
Fig. 1. Experimental design. A total of 5 block trials of the Iowa Gambling Task (IGT) and 6 block trials of the control task (CT) were performed.

Fig. 2. Locations of probes in near-infrared spectroscopy (NIRS). (A) Probe setting with one $3 \times 5$ (probe 2 ) and two $3 \times 3$ (probe 1 ) thermoplastic shells from the frontal view. (B) Two-dimensional topographic map. (C) Estimated cortical regions using the virtual registration method.

Fig. 3. Performance of the Iowa Gambling Task (IGT) by patients with bipolar disorder (BD) and by healthy control subjects during 5 consecutive stages of the task. Error bars indicate standard deviations of the means. There were no differences in performance between two groups.

Fig. 4. Grand-averaged waveforms of oxygenated hemoglobin (oxy-Hb) levels during the Iowa Gambling Task (IGT) and Verbal Fluency Task (VFT) in patients with bipolar 
disorder (BD) and healthy control subjects. PFC, prefrontal cortex; TG, temporal gyrus; OFC, orbitofrontal cortex.

Fig. 5. Areas under the curve for the 6 regions of interest (ROIs) included the prefrontal cortex (PFC), the temporal gyrus (TG), and the orbitofrontal cortex (OFC). (A) During the Iowa Gambling Task (IGT). (B) During the Verbal Fluency Task (VFT).

Patient groups showed significantly decreased oxygenated hemoglobin (oxy-Hb) levels in bilateral OFC and left PFC during the IGT, but no significant changes were observed during the VFT. Data are presented as the mean $\pm \mathrm{SD} ;{ }^{*} p<0.008$.

Fig. 6. Mean changes in oxygenated hemoglobin (oxy-Hb) levels in the left orbitofrontal cortex (OFC) during the Iowa Gambling Task (IGT) in healthy subjects and patients with bipolar disorder (BD).

Fig. 7. Receiver operating characteristic (ROC) curve analyses of the Iowa Gambling Task (IGT) and the Verbal Fluency Task (VFT) in the left orbitofrontal cortex (OFC). AUC, area under the curve. 
Fig.1

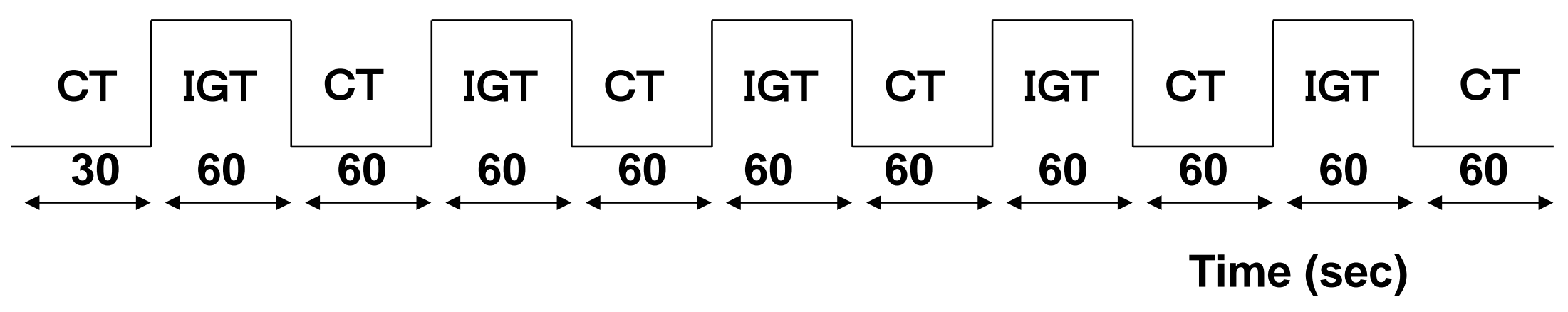


Fig.2A

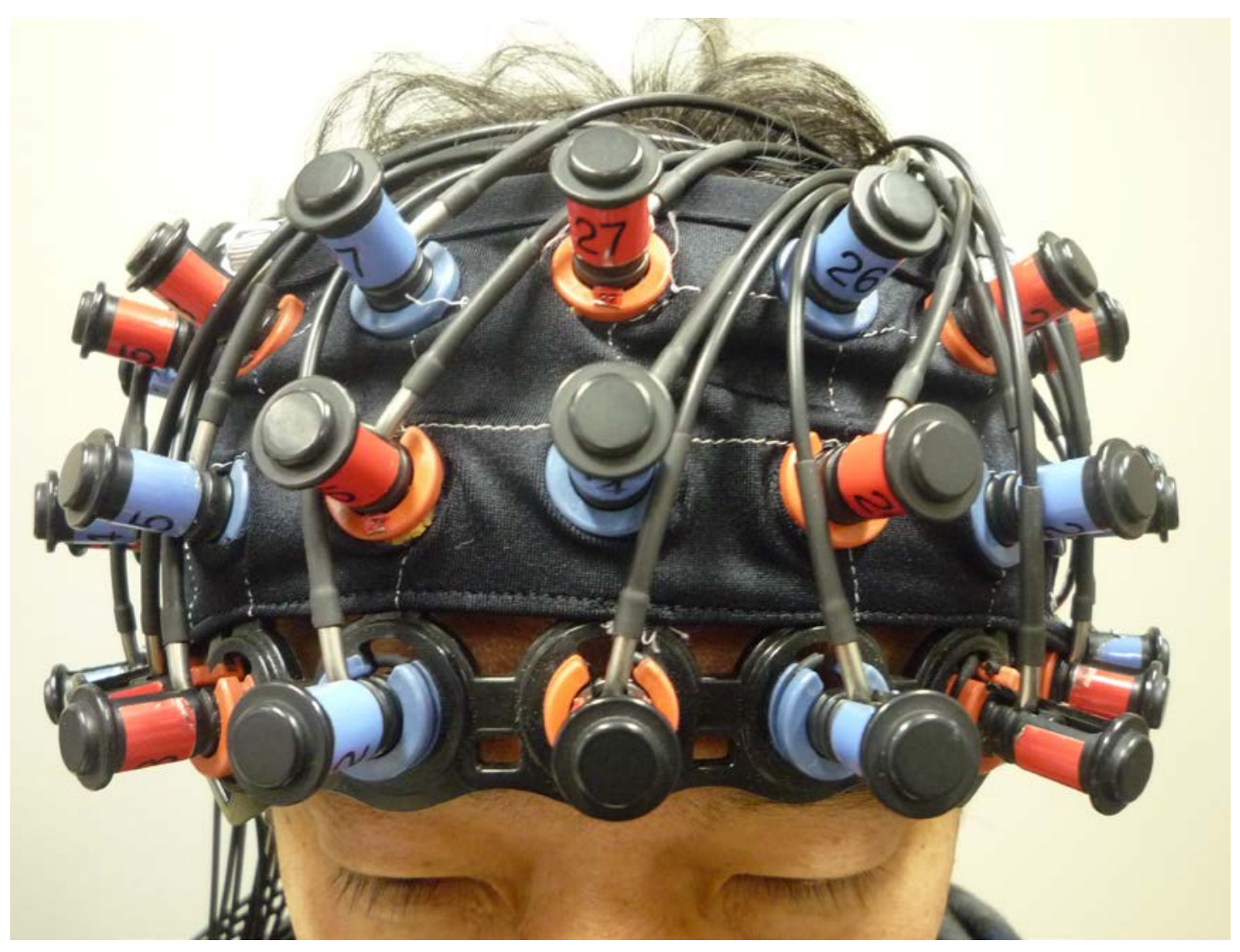


Fig.2C

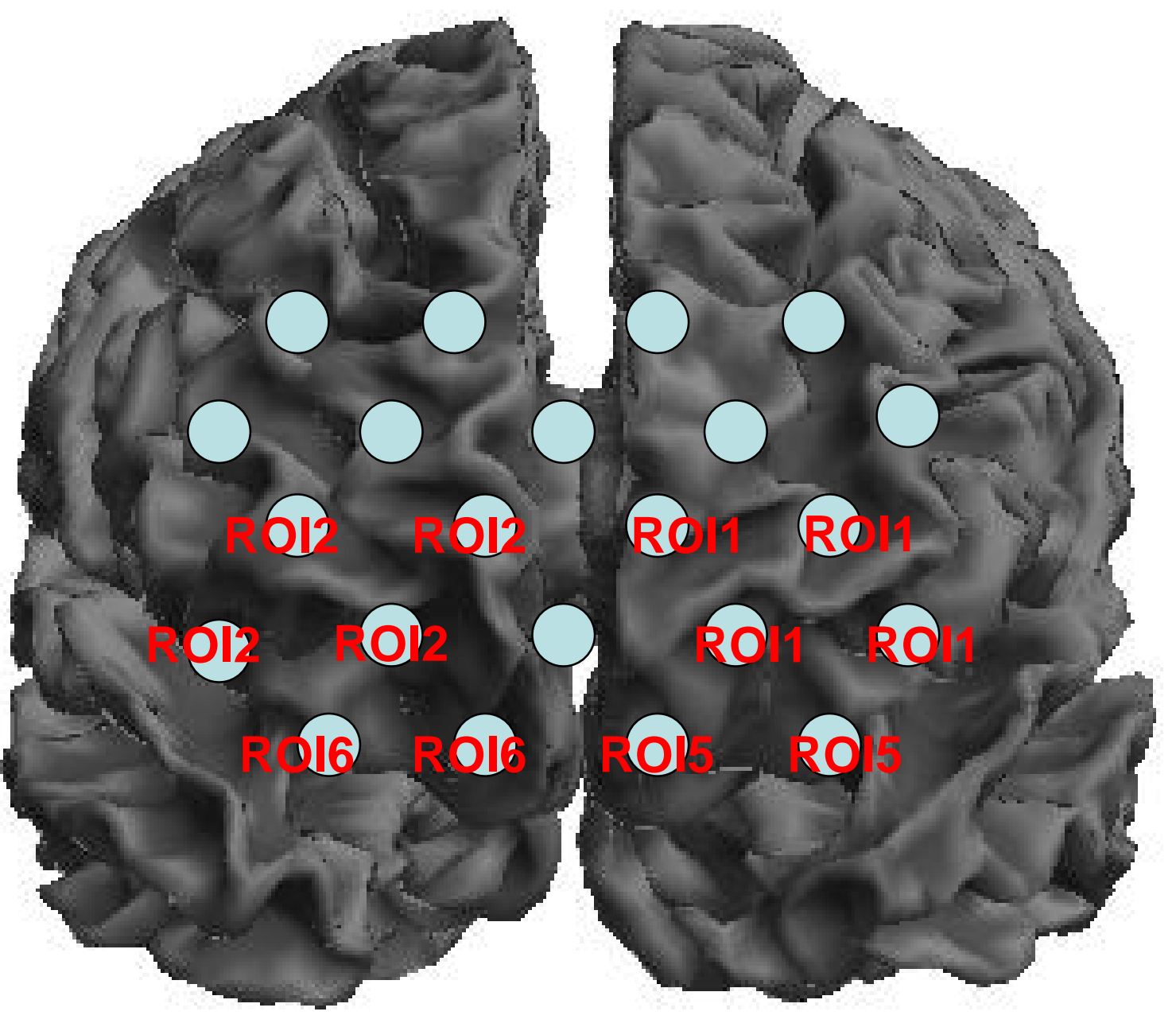




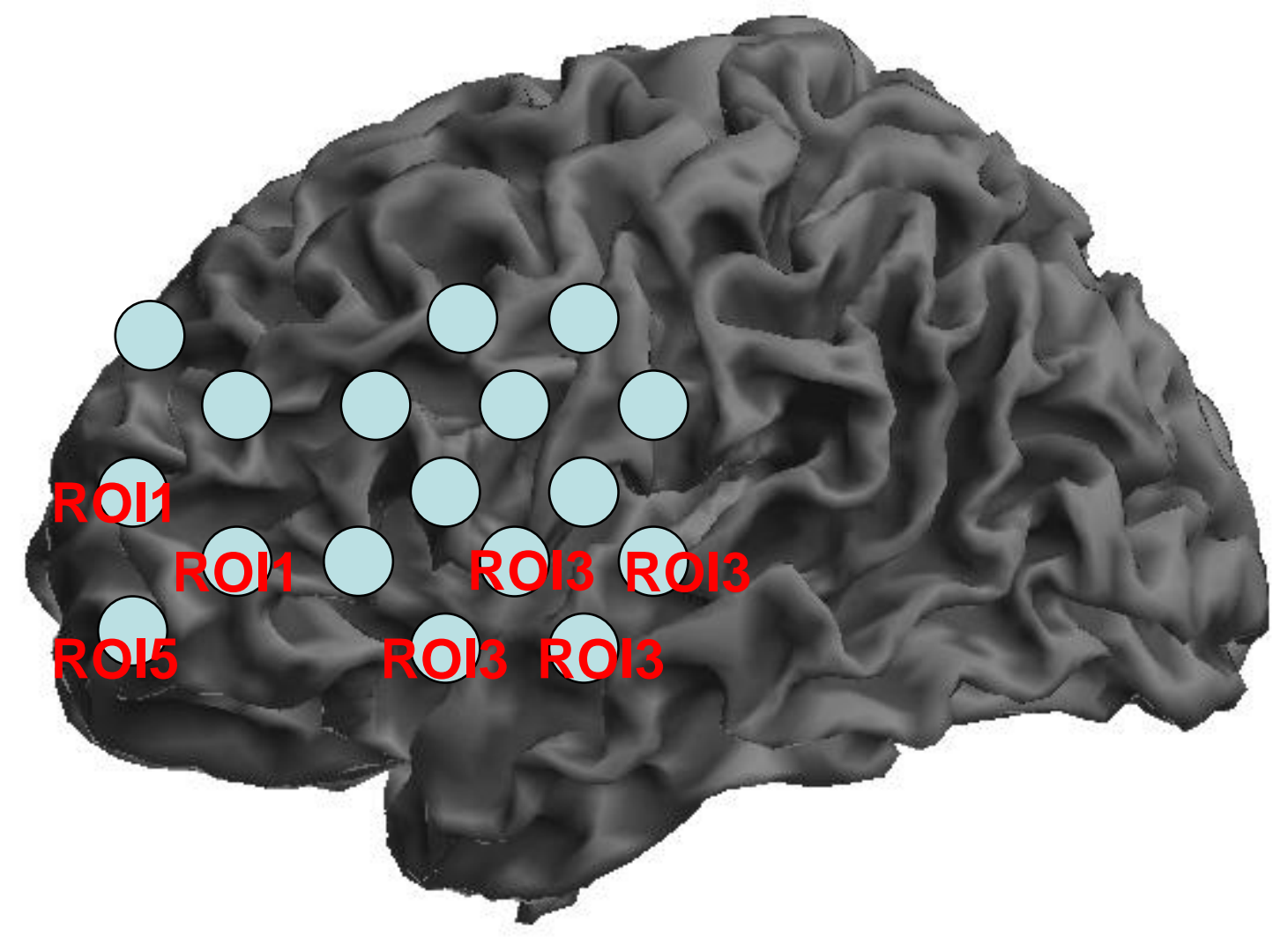




\section{Fig.3}

\section{IGT}

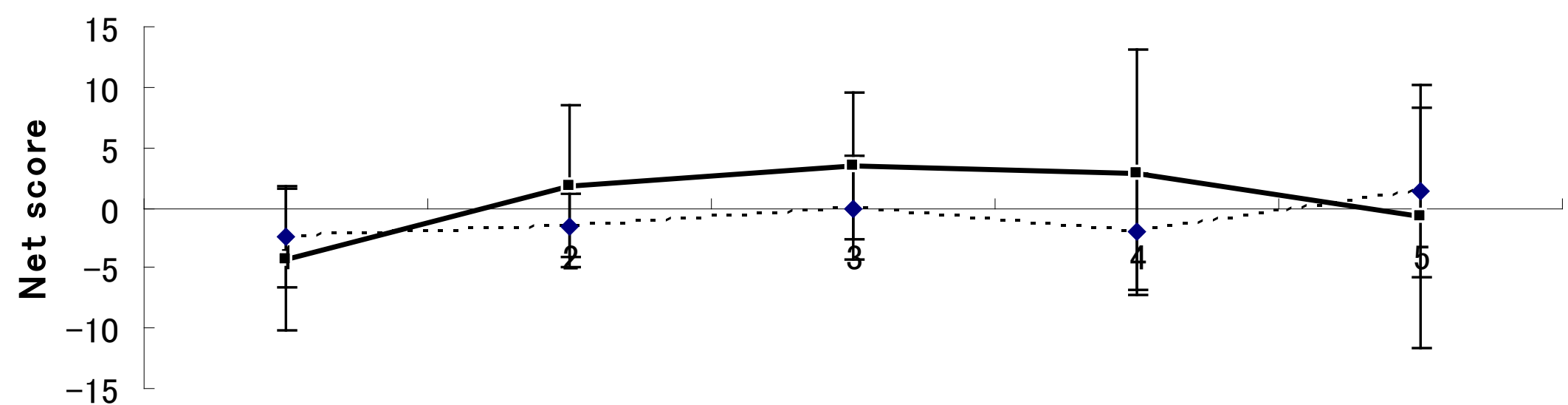

Number of blocks

… BD - control 


\section{Fig.4}

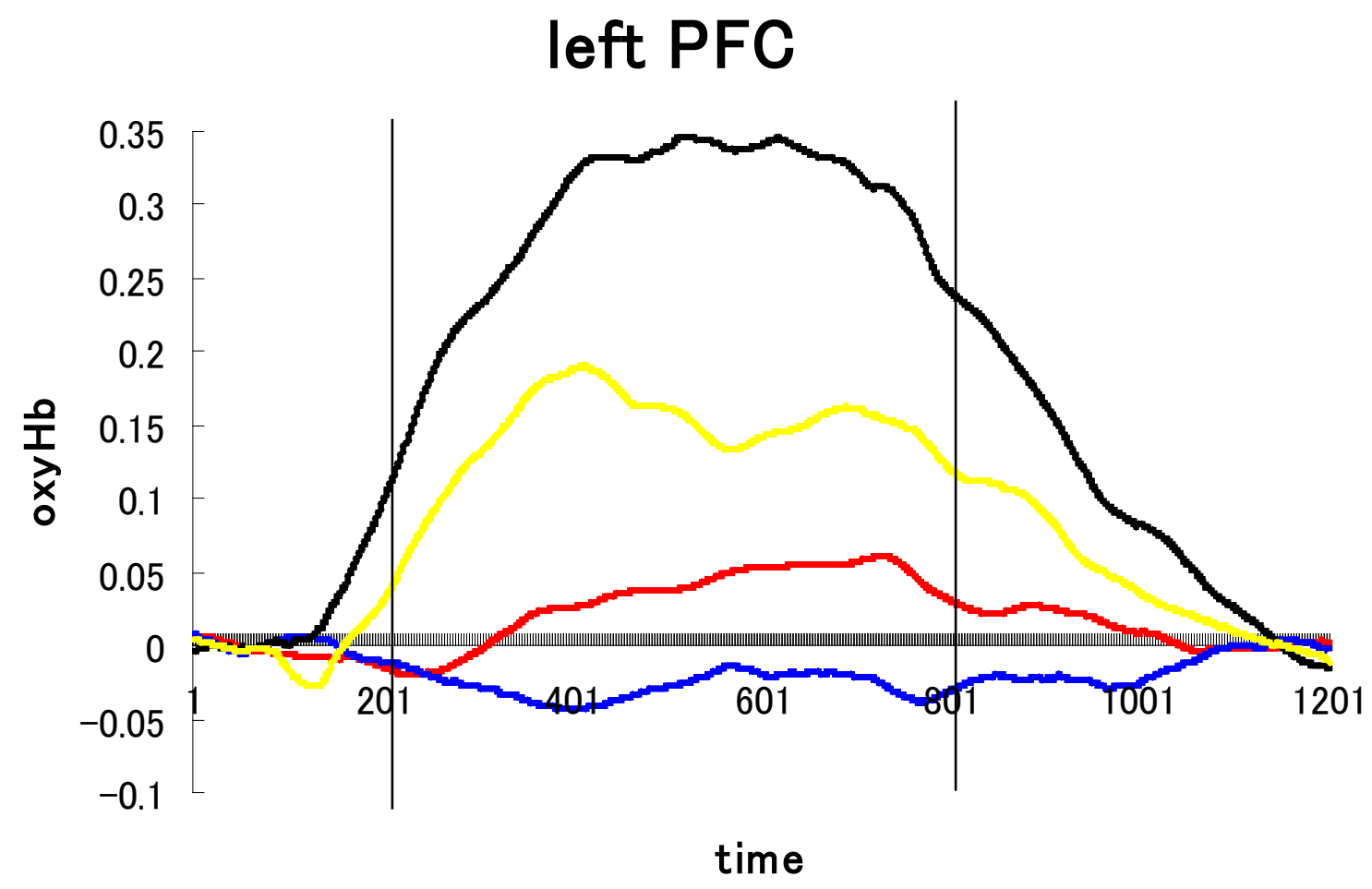

- control(IGT) $-\mathrm{BD}(\mathrm{IGT})-\mathrm{control}(\mathrm{VFT})-\mathrm{BD}(\mathrm{VFT})$ 


\section{right PFC}

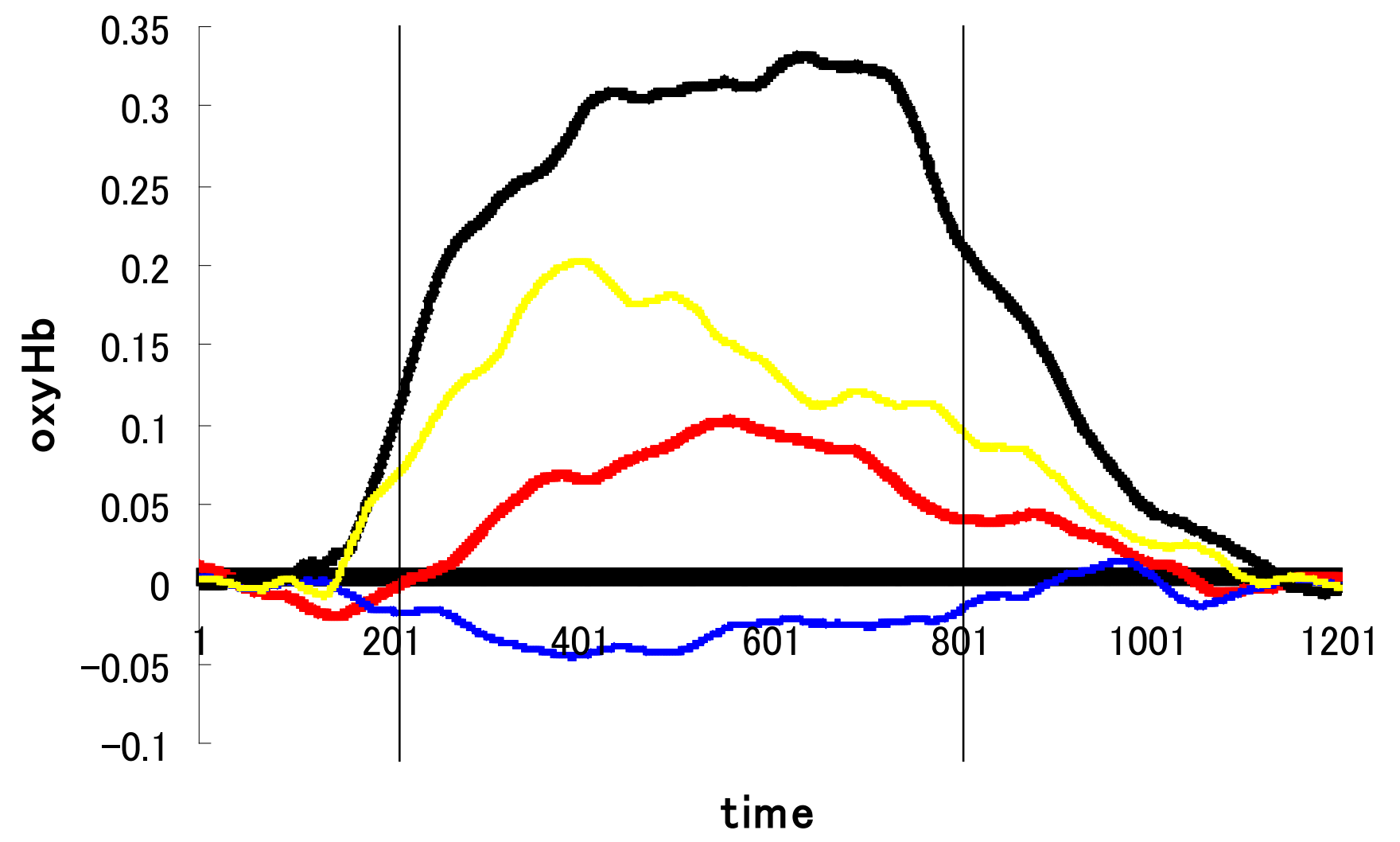


left TG

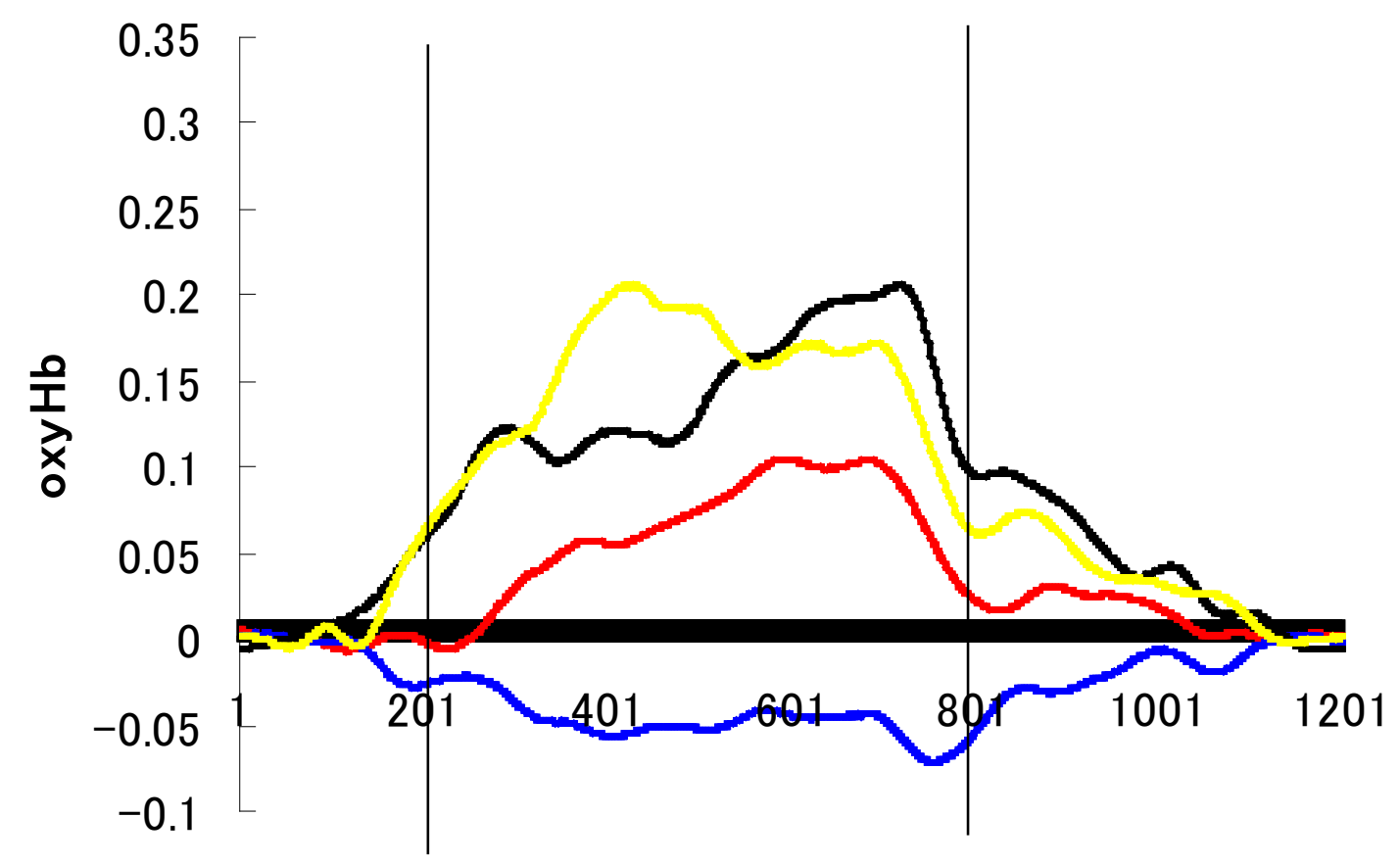

time

- control(IGT) $-\mathrm{BD}(\mathrm{IGT})-$ control(VFT) $-\mathrm{BD}(\mathrm{VFT})$ 


\section{right TG}

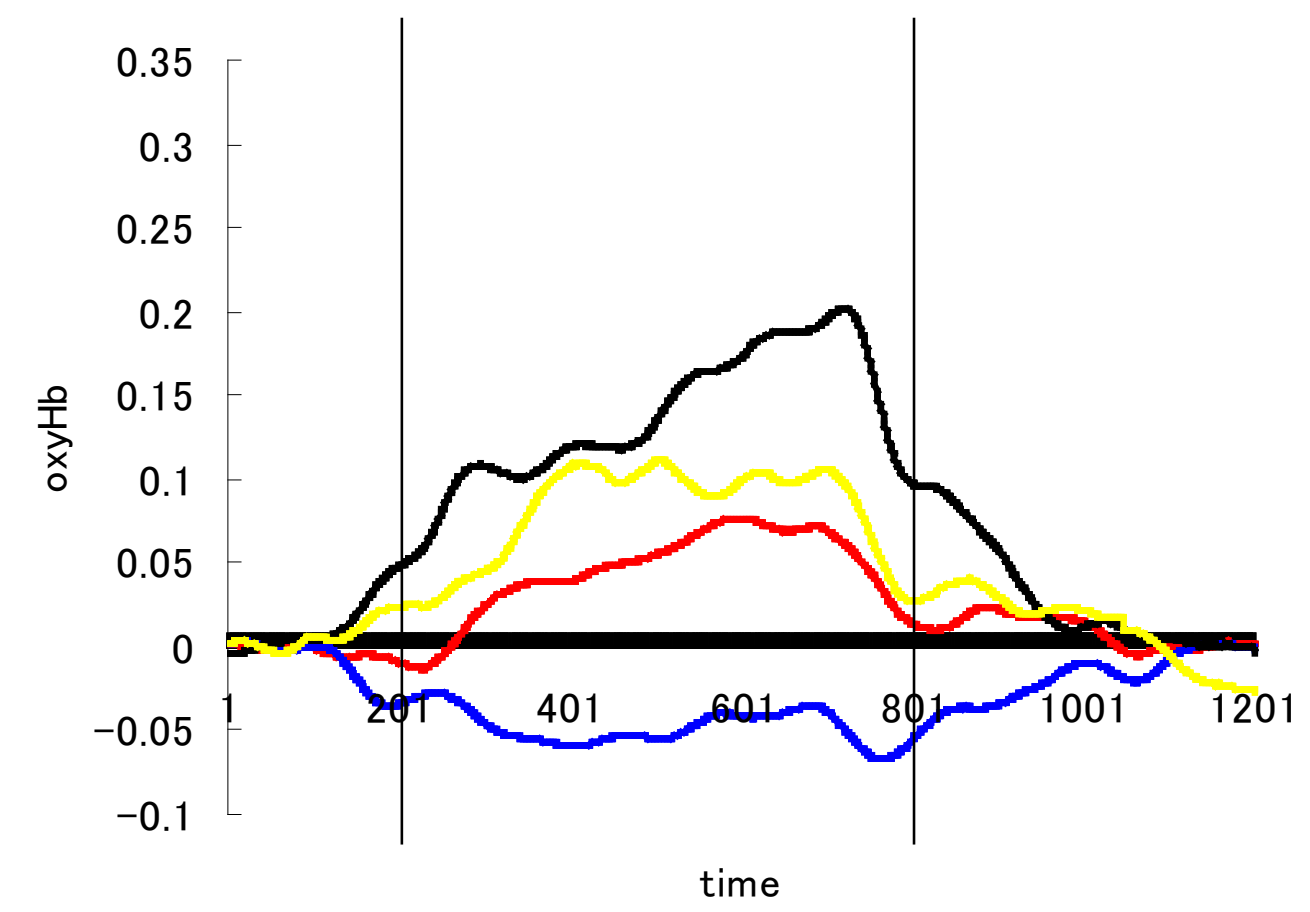

- control(IGT) - $-\mathrm{BD}(\mathrm{IGT})-$ control(VFT) $-\mathrm{BD}(\mathrm{VFT})$ 


\section{left OFC}

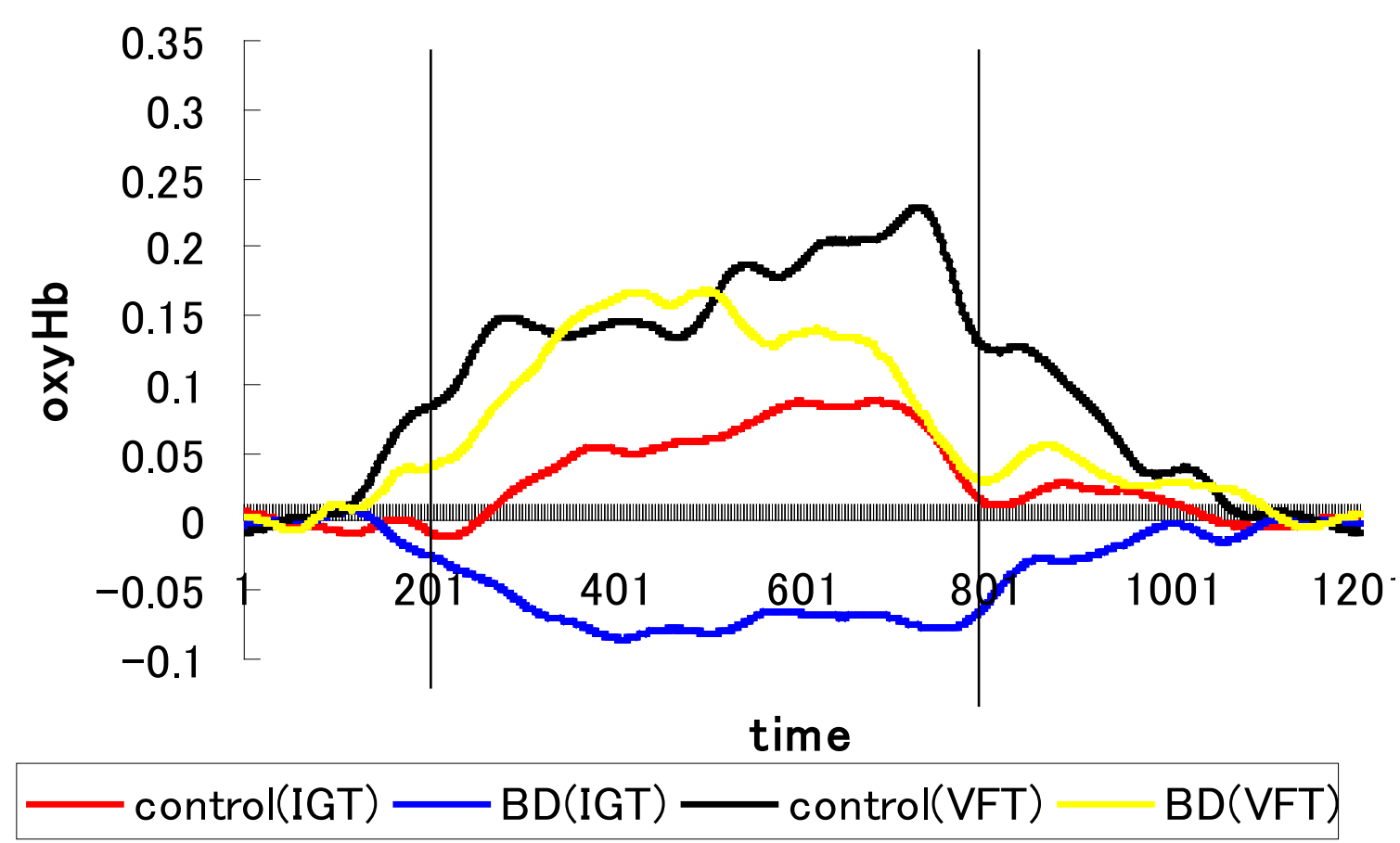




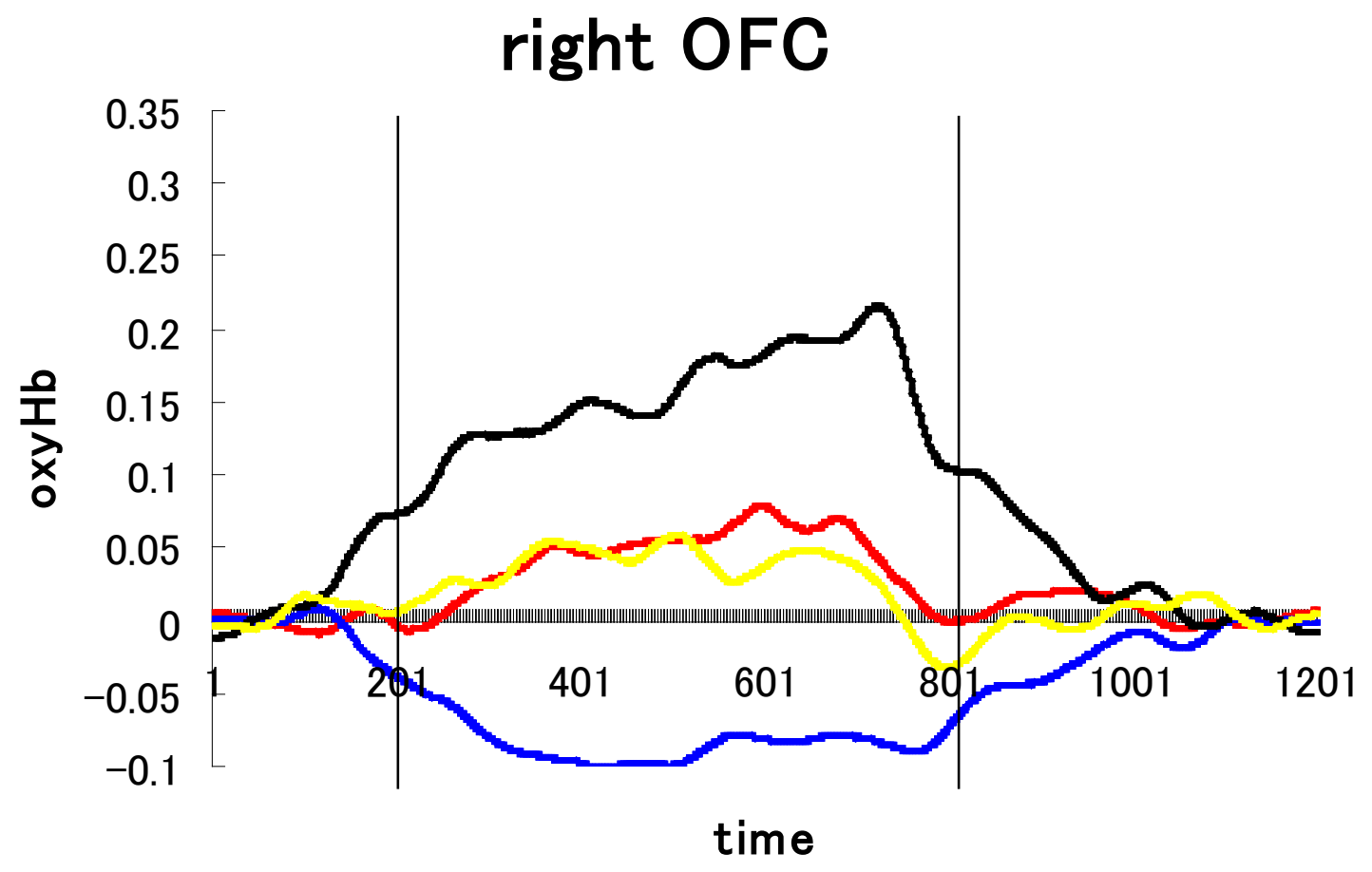

- control(IGT) $-\mathrm{BD}(\mathrm{IGT})-\operatorname{control}(\mathrm{VFT})-\mathrm{BD}(\mathrm{VFT})$ 
Fig.5 (A)

\section{IG T}

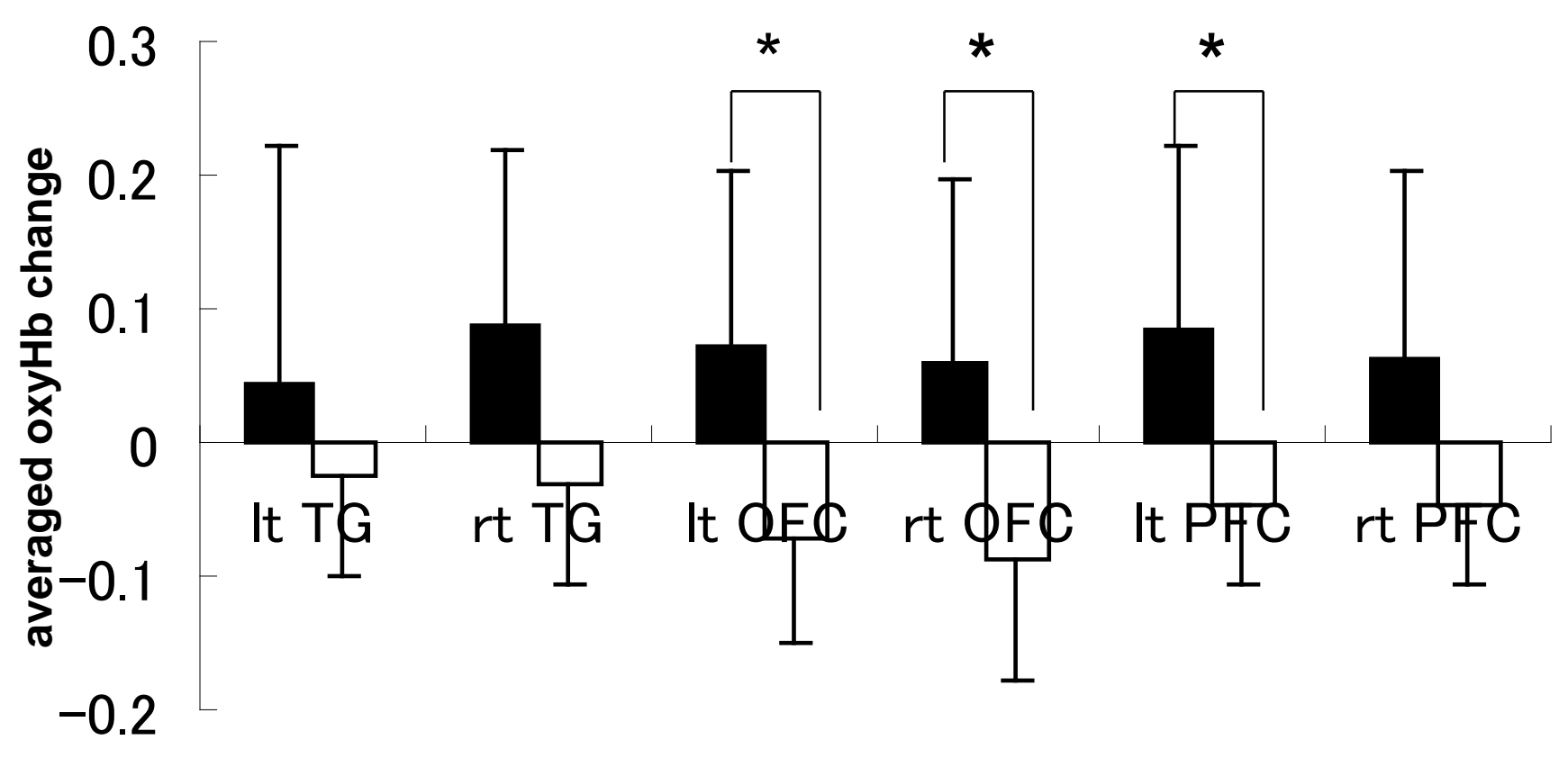

- control $\square$ BD 
Fig.5 (B)

\section{VFT}

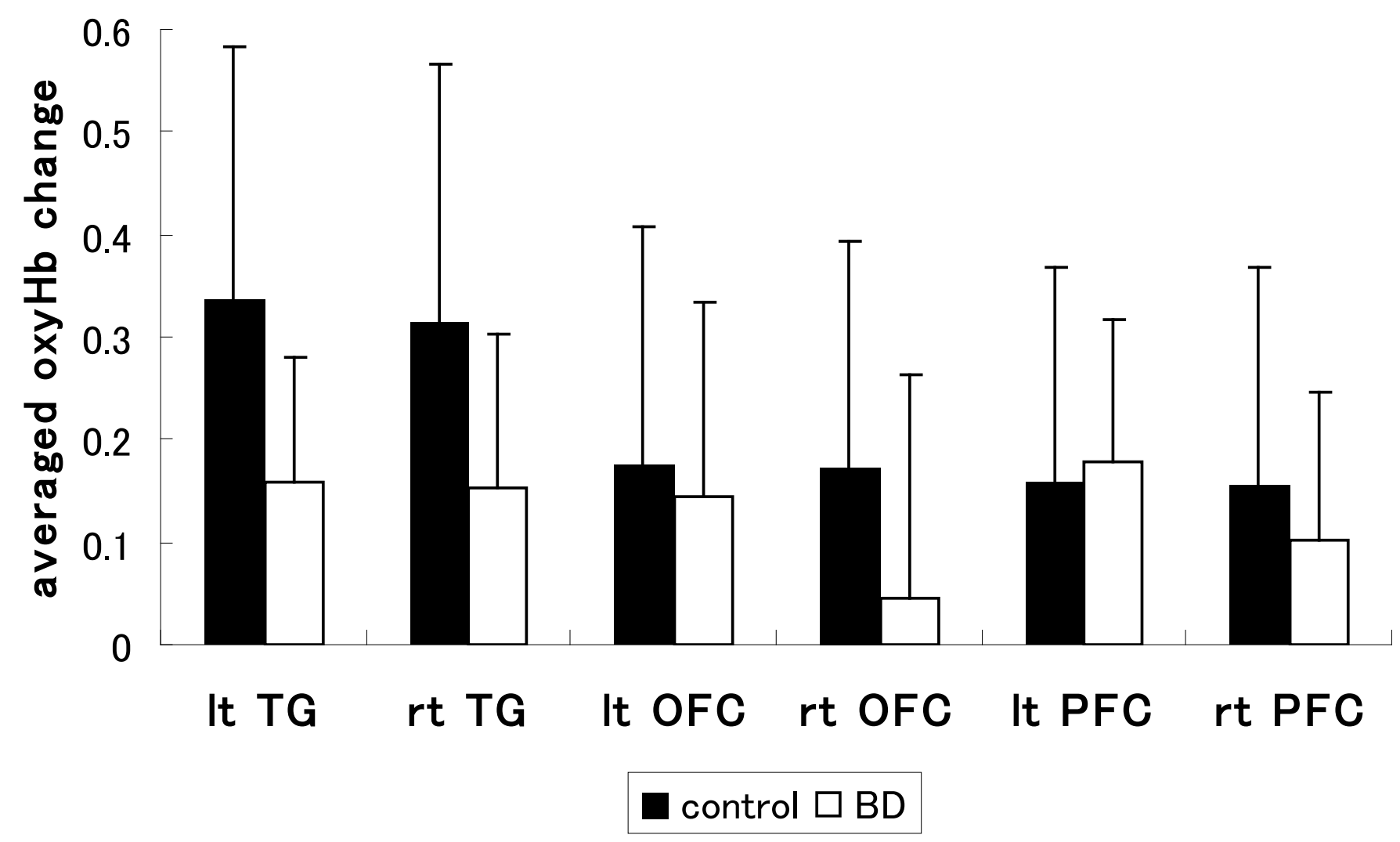


Fig. 6

\section{left OFC}

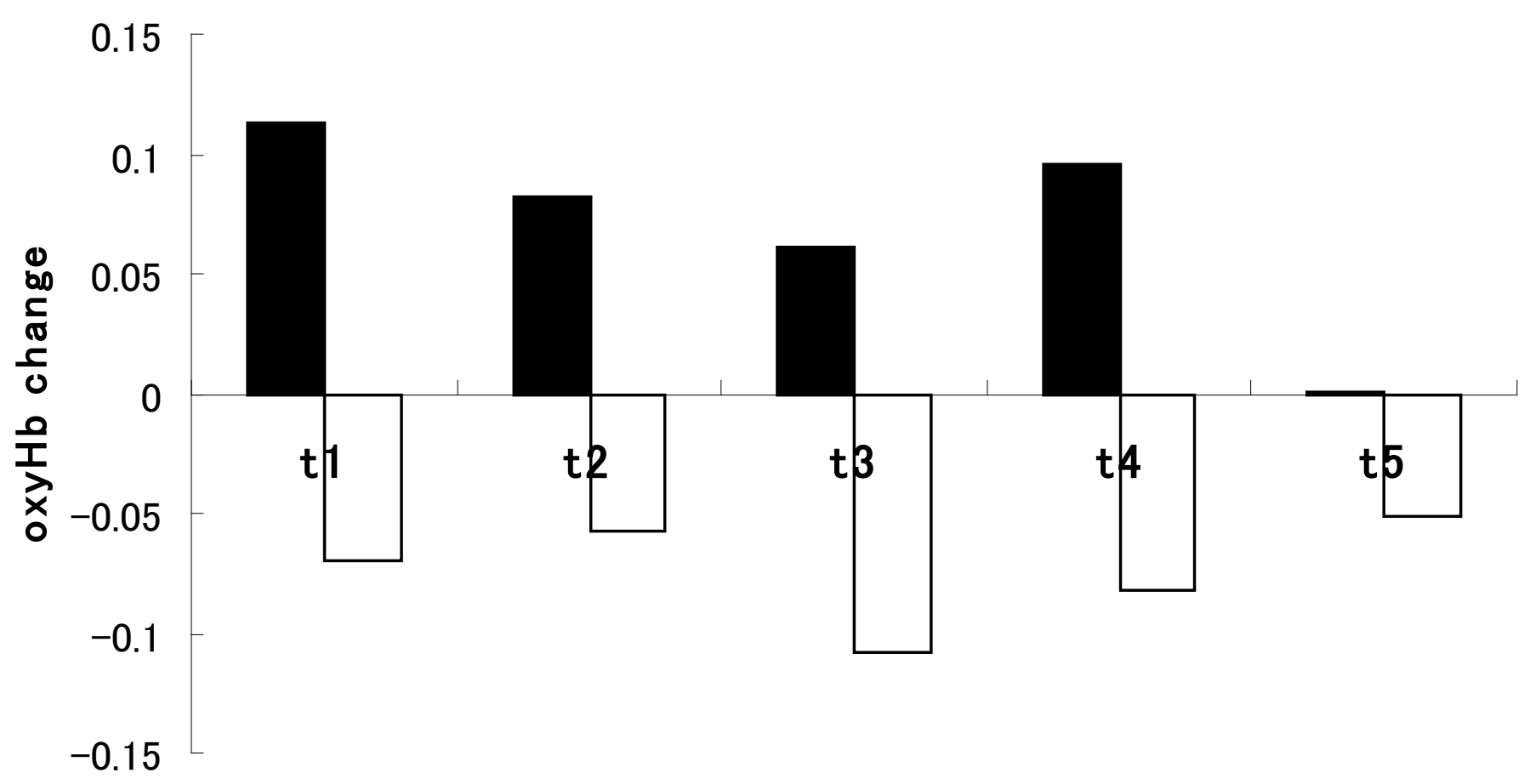

time course

a control $\square \mathrm{BD}$ 
Fig.7

\section{Left OFC}

IGT

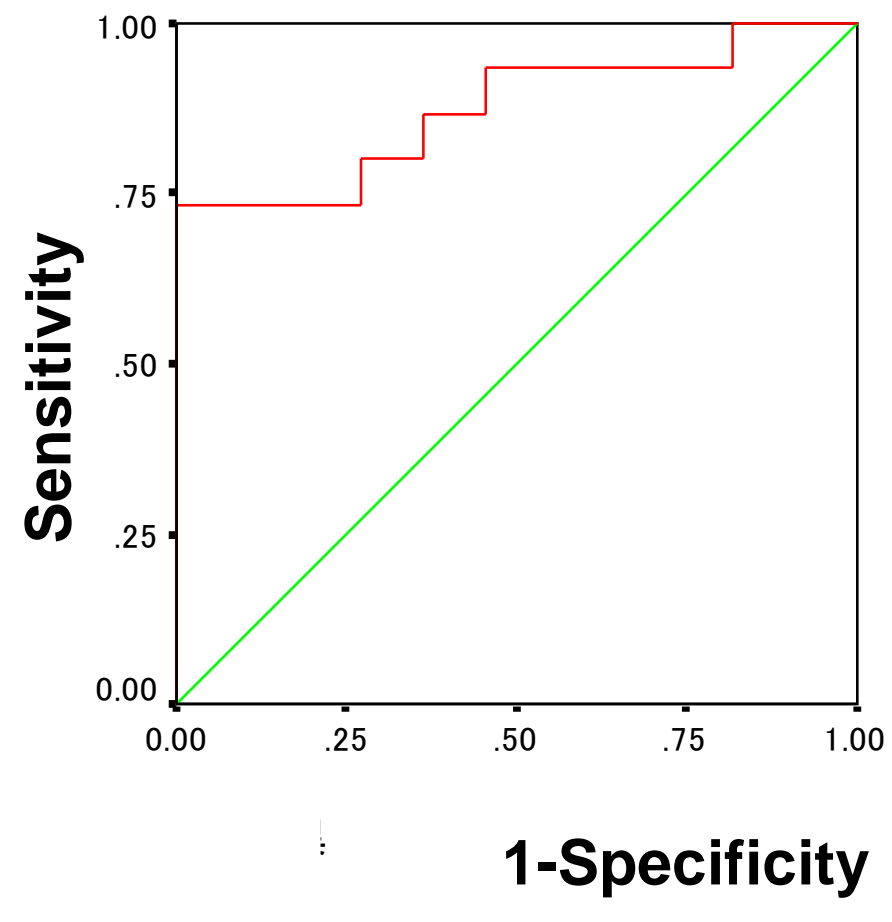

$A \cup C=0.873$
VFT

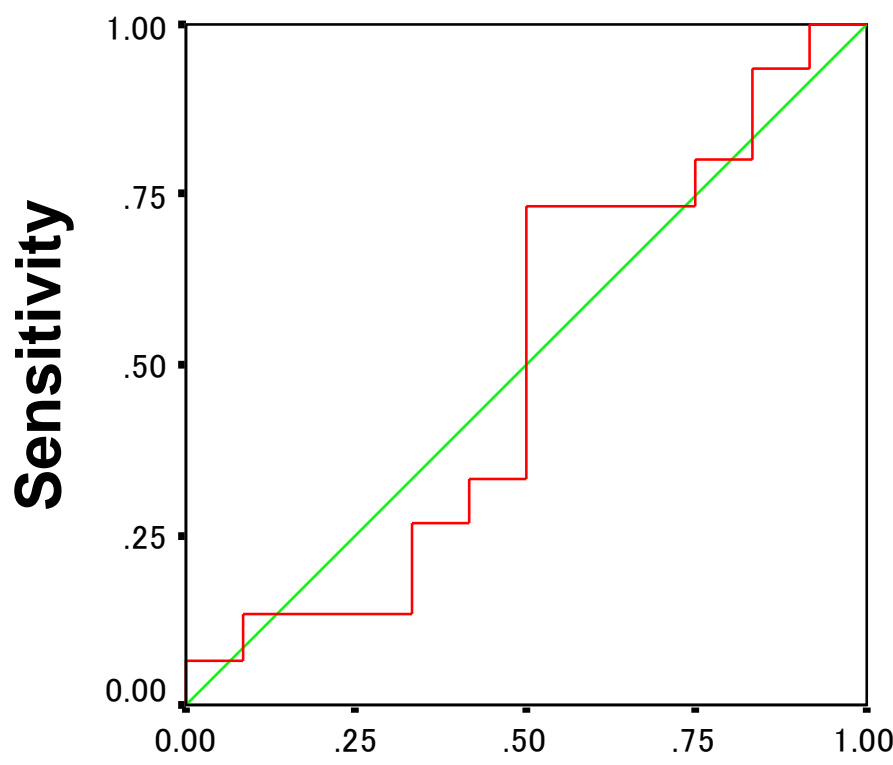

1-Specificity

$A U C=0.500$ 
Table 1 Demographic, clinical and behavior performance of participants.

\begin{tabular}{lccc} 
& $\begin{array}{c}\text { Bipolar depression } \\
(\mathrm{n}=13)\end{array}$ & $\begin{array}{c}\text { Healthy controls } \\
(\mathrm{n}=15)\end{array}$ & Group difference $\mathrm{p}$-value \\
\hline Age (years) & $38.4 \pm 7.3$ & $32.9 \pm 7.7$ & 0.068 \\
Sex (female/male) & $\mathrm{f:m=7:6}$ & $\mathrm{f:m=7:8}$ & 0.705 \\
Edinburgh handedness inventory(\%) & $93.8 \pm 9.6$ & $98.7 \pm 3.5$ & 0.108 \\
Estimated premorbid IQ & $101.5 \pm 9.6$ & $106.3 \pm 9.7$ & 0.201 \\
GRID HAM-D21 total score & $12.2 \pm 6.6$ & $1.3 \pm 1.8$ & $\mathrm{P}<0.001$ \\
YMRS & $2 \pm 2.0$ & $0.3 \pm 0.8$ & 0.011 \\
BADS & $100.1 \pm 12.2$ & $107.3 \pm 11.1$ & 0.116 \\
lowa Gambling Task & $-4.3 \pm 13.0$ & $3.1 \pm 27.8$ & 0.378 \\
Verbal fluency & $8.2 \pm 2.6$ & $11.6 \pm 3.5$ & 0.008 \\
Antidepressive medication & 1 & 0 & \\
Antipsychotic medication & & & \\
Mood stabilizing agents & 8 & 0 & \\
Benzodiazepine use & 13 & 0 & \\
\hline
\end{tabular}

HAM-D, Hamilton Rating Scale for Depression; YMRS, Young Mania Rating Scale; BADS, Behavioural Assessment of the Dysexecutive Syndrome 


\section{Table 2 Area under the curve (AUC)}

right TG left TG right OFC left OFC right PFC left PFC

$\begin{array}{lllllll}\text { IGT } & 0.841 & 0.769 & 0.870 & 0.873 & 0.877 & 0.867 \\ & & & & & & \\ \text { VFT } & 0.718 & 0.728 & 0.612 & 0.500 & 0.574 & 0.446\end{array}$

IGT, lowa gambling task; VFT, verbal fluency task

TG, temporal gyrus; PFC, prefrontal cortex; OFC, orbitofrontal cortex 
Table 3 Results of each physiological variable.

\begin{tabular}{lllllll} 
& & $\mathrm{t} 1$ & $\mathrm{t} 2$ & $\mathrm{t} 3$ & $\mathrm{t} 4$ & $\mathrm{t} 5$ \\
\hline LnLF & control & $5.24 \pm 0.77$ & $5.09 \pm 0.80$ & $5.23 \pm 0.82$ & $4.96 \pm 0.72$ & $5.49 \pm 0.92$ \\
& $\mathrm{BD}$ & $5.47 \pm 0.99$ & $5.09 \pm 1.04$ & $4.94 \pm 0.66$ & $5.11 \pm 0.77$ & $5.19 \pm 0.92$ \\
\hline LnHF & control & $4.73 \pm 1.08$ & $4.8 \pm 1.15$ & $4.71 \pm 1.18$ & $4.79 \pm 1.05$ & $4.73 \pm 1.06$ \\
& BD & $4.62 \pm 1.05$ & $4.46 \pm 1.1$ & $4.42 \pm 1.24$ & $4.48 \pm 1.13$ & $4.43 \pm 1.32$ \\
\hline LF/HF & control & $2.84 \pm 2.90$ & $2.04 \pm 1.82$ & $2.28 \pm 1.61$ & $1.63 \pm 1.10$ & $3.00 \pm 2.15$ \\
& BD & $2.9 \pm 2.95$ & $2.26 \pm 2.10$ & $1.59 \pm 0.96$ & $1.8 \pm 0.97$ & $1.89 \pm 1.16$ \\
\hline
\end{tabular}

LF, low-frequency $(0.05-0.15 \mathrm{~Hz}) ; \mathrm{HF}$, high-frequency $(0.15-0.40 \mathrm{~Hz})$ ; LF/HF, ratio of LF power to HF power.

Values are the mean \pm S.D. 
Table 4 Correlation between HAM-D score and oxy-Hb.

HAM-D vs IGT right TG left TG right OFC left OFC right PFC left PFC

\begin{tabular}{lrrrrrr}
\hline$r$ & -0.338 & -0.089 & -0.456 & -0.465 & -0.39 & -0.384 \\
$P$ value & 0.078 & 0.654 & 0.022 & 0.017 & 0.04 & 0.044
\end{tabular}

HAM-D vs. VFT

\begin{tabular}{lrrrrrr}
\hline$r$ & -0.439 & -0.366 & -0.154 & -0.235 & -0.187 & -0.005 \\
$P$ value & 0.02 & 0.055 & 0.444 & 0.249 & 0.341 & 0.98
\end{tabular}

HAM-D, hamilton rating scale for depression; IGT, lowa gambling task; VFT, verbal fluency task TG, temporal gyrus; PFC, prefrontal cortex; OFC, orbitofrontal cortex 